\title{
Gemming: Architectonics of Facets
}

\author{
Daniel Baerlecken \\ Georgia Institute of Technology, USA \\ daniel.baerlecken@arch.gatech.edu \\ Sabri Gokmen \\ Georgia Institute of Technology, USA \\ sabrigokmen@gatech.edu
}

\begin{abstract}
This paper will articulate on how the intrinsic principles of gem making could be applied in architectural form finding. It will speculate that the technical accumulation of expertise and knowledge in this crafty technique could be implemented algorithmically. This robust and efficient process could provide solutions for architectural design problems. It will conclude that faceting, as we can observe it in jewelry making and lamp design, produces facets that are not just reacting to an underlying geometry, but also add expression and articulation to an object.
\end{abstract}

Keywords: Planar facets, Tiffany lamps, Color, Digital design.

\section{Introduction}

The paper "Gemming" presents a research and design project that focuses on faceting of surfaces as a method to design intricate, highly articulate surfaces. The process of faceting is not so much understood as an optimization process of a double-curved surface in order to match the original intent of the designer (J. Glymph et al., 2004), but more as a process of obtaining design knowledge that allows enriching the quality of the surface. Gemstones are facetted in order to enhance their optical performance so that the different angles used for each facet can create reflections and refractions. For this effect gem cutters use an analog faceting machine that consists of a grinding wheel working with a mast and a quill. In order to achieve the same effect digitally most approaches extract points on a Nurbs surface with different distribution, which will be discussed in detail. Furthermore we can differentiate between flat facets, that follow a given surface curvature as well as facets that transform a surface within a certain range and apply another set of 3-dimensional facets to it.

In order to develop computational strategies to create facets and other variations of faceting we look at triangular facets, quadrilateral facets, diamond shaped facets, hexagonal facets, voronoi-based faceting (M. Stavric + A. Wiltsche, 2011), natural faceting in quartz crystals, $n$-sided faceting in jewelry making (Swarovski) and mosaic facets (Tiffany lamps).

In Swarovski crystals that are cut and shaped into dolphins, gorillas, bears and swans, we can see how the geometry of facets transforms and adapts, as the operation transitions from various parts of the modeled animal. Sometimes a hexagonal system is used to articulate the back
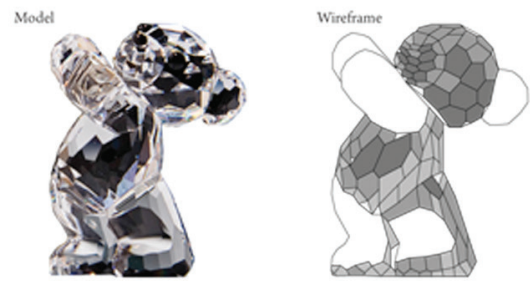

Pentagonal Facets

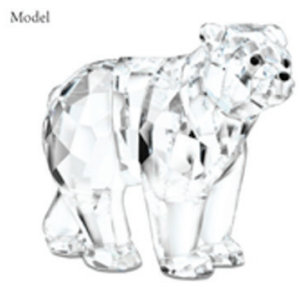

Tetragonal Facets

Figure 1: Swarovski Crystals.

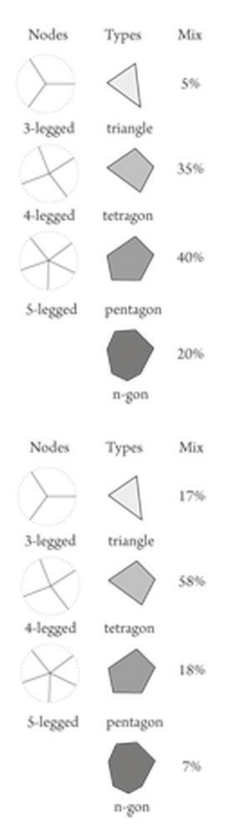

225 of a lion, while a triangular system is used for the mane to add variation to the design. If we think about the digital consequences of these transformations, it means that on one hand we have an underlying system of points reacting to the surface curvature, while the type of facets is determined by a design decision creating pleasing results. By studying these concepts, the paper will present different faceting outcomes based on different point morphologies on a surface. This is investigated in an algorithmic approach towards converting complex surfaces into planar polygonal tiles. 
In the following section three approaches are presented that traverse through the point information of a curved surface generated by the designer. The approaches include a plane intersection method, a Boolean splitting method, and a population method that we call "gemming". The first algorithm operates using intersections of three planes to define facet points. The second approach uses planar solid geometries for Boolean operation. The final approach recognizes the curvature of the surface and converts it into approximated planar tiles (Cohen-Steiner et al., 2004, Wenping et al., 2008).

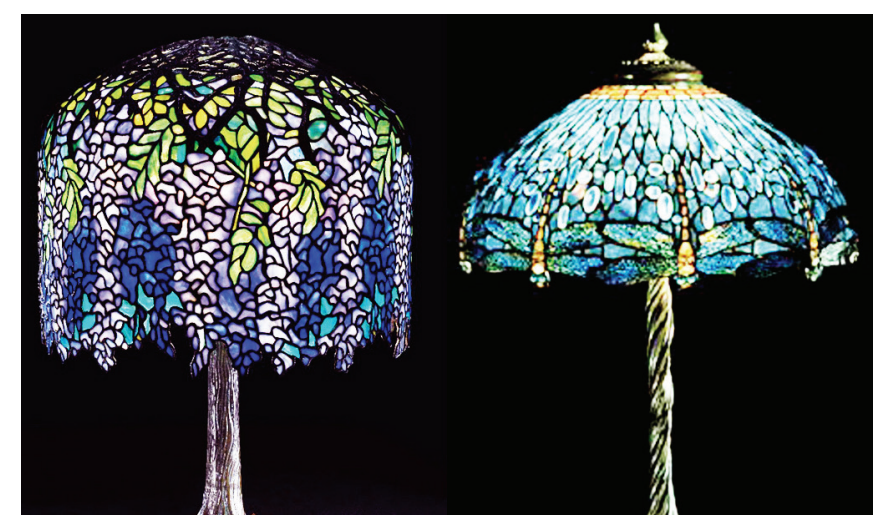

Figure 2. Tiffany lamps.

The next stage of our implementation is the coloring of these surfaces, which will be based on visual and lighting angles that are defined by the designer. This way the tessellation shows variations and gradations of various coloring effects that will enhance the design intent. In the lamp designs from Louis C. Tiffany we can see the use of multi-colored flashed, opalised and streaky mosaic tilings. Tiffany produced paintings in glass (Koch, 1975), which are not attempting to integrate structure and ornament fully. The leaded seams are actually part of the expression of the glass painting. The seams thicken, bifurcate and bundle to create a network of lines to enhance the glass painting. Koch points out that the integration of structure and ornament has been accomplished later in the glass works of Frank L. Wright as a logical outcome of the glass experiments of Tiffany.

\section{Plane-Plane-Plane Intersection}

On our first attempt we revisited a common approach to get subdivision through the construction of planes tangential to the surface. This approach follows the generation of an arbitrary or constrained point cloud over a surface to extract surface normal directions. Using these points and normals of the input Nurbs surface we first compute a Deleunay triangulation to extract adjacency of nodes. The triangles store the vertices with the surface normals that will be later used to compute the intersection point of the planes. These plane points are stored on each triangle that is then used to generate flat planar surfaces (Fig.2).

Although this is the mathematically most accurate method to construct the planar tangential surfaces the algorithmic implementation poses certain problems when the input surface is too complex or the normal distribution and directions are not consistent.

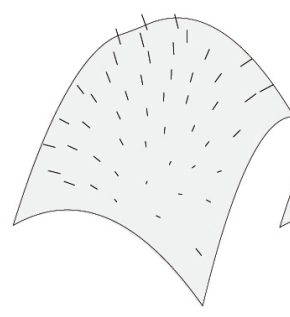

input surface + point cloud

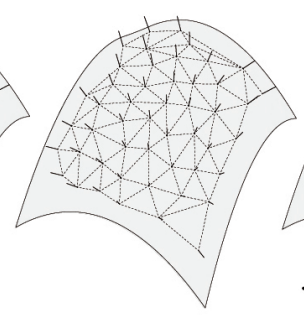

triangulation

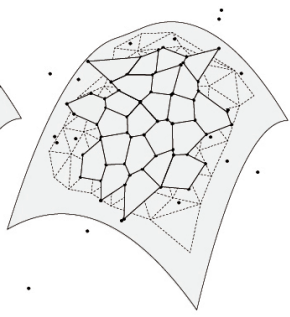

facets
Figure 3: Diagrams showing the implementation steps of plane-planeplane intersection. The mathematically calculated points that are not consistent are shown on the right side.

In many cases the intersection points of the planes might occur outside of the triangles or they might not even be found due to parallel normals. These problems cause the algorithm to perform unstable surface constructions at low Gaussian curvatures. This problem can be fixed by using certain optimization methods such as offsetting normal planes (Zimmer et al., 2013) or edge offset meshes (Pottmann et al., 2007) or using P-Hex meshes (Wenping et al., 2008).
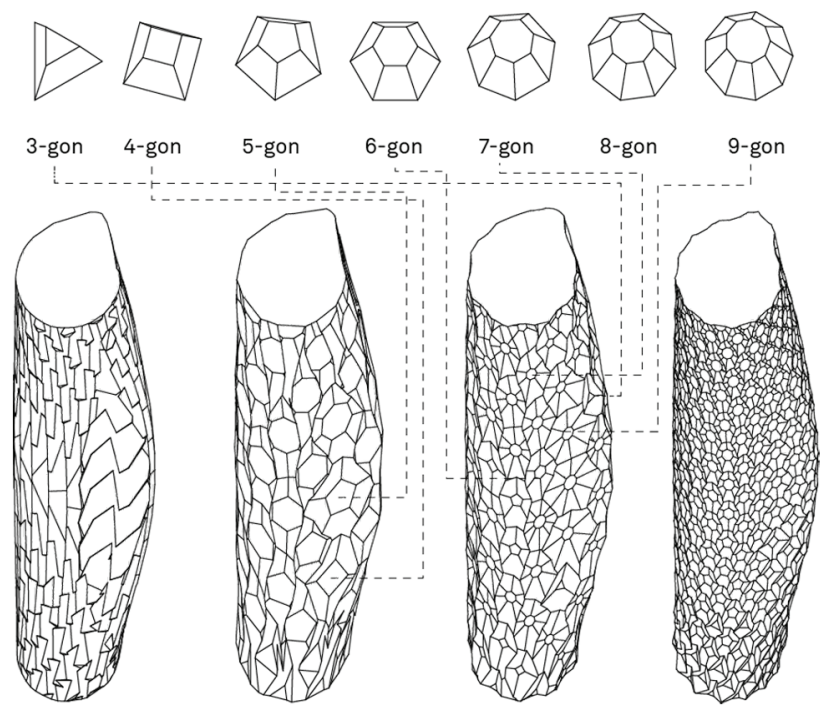

Figure 4: Diagrams showing various iterations of the Boolean method. This method enables the user to populate n-gon shapes over a Nurbs surface

Although such methods provide an optimized mesh planarization, the variations of offset causes the geometry to be locally adjusted or the topology of the tiling to change based on the curvature. Another solution could be found through mesh optimization methods that are computationally expensive methods (CohenSteiner, 2004). Although this automated process maintains overall features and symmetries of the geometry, it gives less control to the user as the conversion is executed through mathematical approximation. In many optimized solutions the output geometry contains irregular concave polygons that disrupt topological consistency and patterning over the surface. 

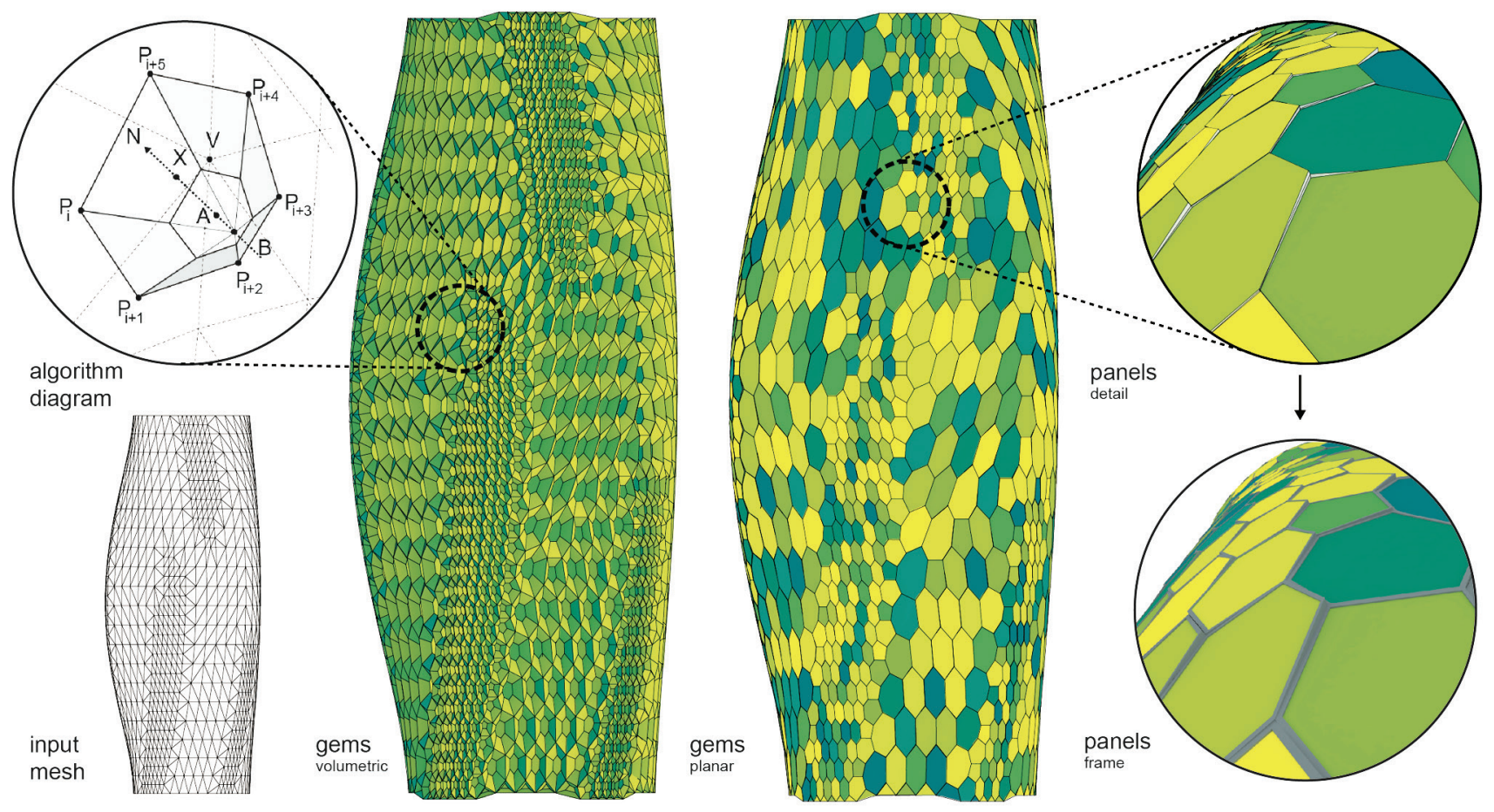

Figure 5: Diagrams showing the conversion of the mesh surface into planar components. The length of the polygons are used as a thickening parameter for panel computation.

\section{Boolean Method}

The second approach is based on a series of Boolean operations that are applied to a surface. A hexagon grid is created on a surface. Based on these points a circle is created that is perpendicular to the normal vector at each of the extracted points on the surface. In order to create a multitude of different polygonal polylines the circle is divided into different amounts of points ranging from 3 to 10 points. This division can be based on the random point generation or it can be based on another rules set. The polygon is then created through these points on a circle and the outer polygon that point going to shape is moved inwards in direction of the normal vector. The inner polygon is scaled to 50 $\%$ of its original size. In a next step the outer polygonal and the inner one are lofted, which creates a tapering volume. The tapering allows to overcoming the problem of the polygonal edges and to change from convex to concave curvature. In a last step each solid is subtracted from the given surfaces, which creates the final faceted surface.

This approach produces a huge variety of facets and enables the designer to intentionally highlight areas of the façade design. Varying the size of the Boolean n-gon, the amount of n-gon, the distribution of $n$-gons and the tapering of each $n$-gon has a drastic impact on the emerging qualities of the surface ranging from jagged to almost homogeneous appearance (Fig.4).
The technical implementation has proven to be unreliable, because the Boolean operations often fail in Rhinoceros / Python. That makes the execution of a script only possible to a certain extent and leaves the last step, the Boolean, to manual modeling in a trial and error procedure.

\section{Curvature-based Gemming}

In our final approach we have generated a mesh based surface approximation that combines plane intersection and $\mathrm{n}$-gon panelization without requiring any Boolean operations. We start with any triangulated mesh surface to compute polygonal regions $(\mathrm{P})$ that surround a mesh vertex (V) (Fig.5). Using the mesh connectivity, we compute an average surface normal $(\mathrm{N})$ located at the center of the polygon $(X)$ that will be used to generate flat panels. We use the normal to define two sets of points. The first point $(A)$ defines the location of the inner plane of the tessellation while the second point defines an extrusion point (B) that is used for plane ${ }^{3}$ intersection. These points are informed by the length of the polygons that define the depth of the panels as a controllable parameter.

To find the facet points we traverse the outer polygon vertices $\left(P_{i}\right)$ and look for the plane ${ }^{3}$ intersection among $\lambda\left(P_{i}, P_{i+1}, B\right)$ and $\lambda\left(P_{i+1}\right.$, $\left.P_{i+2}, B\right)$ and Plane $(A, N)$. After this operation is done we sort the facet points to define the inner 

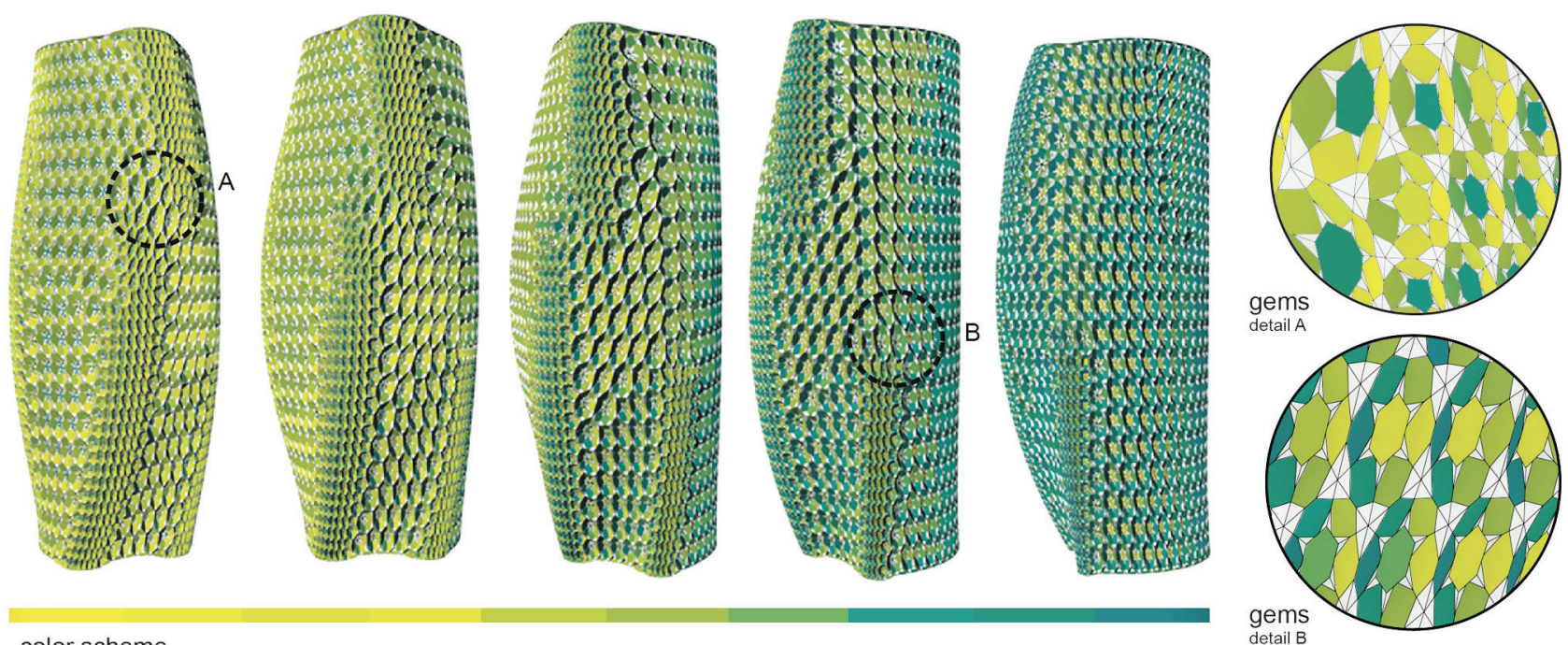

color scheme

Figure 6: Diagrams showing a color method that blurs the edge of facets

$\mathrm{n}$-gon and the side panels that are both flat pieces. We repeat this process for all the surface polygons. Using outer polygons for planarization creates consistent edge connectivity and planar approximation using volumetric panels. In addition, the algorithm follows the input mesh geometry efficiently and produces $\mathrm{n}$-gon shapes that repeat and create surface guided tessellation patterns.

We have tested our gemming approach on various complex surfaces to test the performance of our algorithm over

pinched, double curved, convex and concave surfaces. The results show that the algorithm is able to maintain consistency while following the input mesh curvature and connectivity. There are various parameters that could be controlled during this process such as the depth of the panels and the angles of the side panel faces. When the depth parameter is ignored the algorithm approximates the surface into planar convex polygonal tessellations (Fig.5) . However these facets do not maintain edge connectivity due to the simplicity of the implementation, yet they create a continuous surface specific ornamental pattern. For constructability the irregularity of the edge conditions need to be compensated by the joining elements. Like the edge conditions in Tiffany lamps, these discontinuities could be compensated by using supporting material that enable different intersections with the flat surfaces on both sides.

\section{Coloring - Sharpening}

As an extension to our construction algorithm we have added a further step to add color to the computation based on the angle of the faceted surfaces. Using gradients of multiple colors we assign surfaces facing the same direction the same colors. This way the overall surface acquires a gem-like quality where the coloration emphasizes the angles of the facets as well as gives the surface geometry a colorful pattern. We have tested the coloration on volumetric facets as well as planar facets where the thickening parameter is set to zero. While the former produces a continuous pattern, the latter creates an ornamental arbitrary color distribution due to the surface angles (Fig.5-6). Furthermore the volumetric panels could be used to highlight the directionality of the surface using color that can give the design an opulent and textured effect.

\section{Coloring - Edge Blurring}

Whereas color sharpening tries to enhance the visual quality of each facet by applying color to each facet, the edge blurring method tries to create an additional reading of another pattern that operates against the individual facet (Fig.6). Color is applied around the nodal points, which unifies parts of different facets. This approach blurs the edges. While the first approach towards color can be seen in analogy to Tiffany lamps, this approach follows a technique that we can see in Frank L. Wright's treatment of surfaces, for example in his project for Unite Temple in Oak Park, where Wright warps color and lines around corners of wall and balustrades. This allows multiple readings and creates visual continuities, where no geometrical continuity exists.

\section{Conclusion}

In this paper we have presented three approaches that can convert surfaces into planar facets that can facilitate various coloring and patterning effects. Among these methods we have consider the gemming approach to provide control over surface input, parameterization of panels and coloring based on the angles of the generated facets. Although gemming provides an efficient tool for design further research on construction detailing and optimization of joints would be necessary. We believe that following a design oriented approach using the tectonic qualities of Tiffany lamps and F. L. Wright's edge operations could lead to interesting aesthetic outcomes for surface planarization. Compared to other computational methods that focus on 
optimized solutions, we give the design intent a higher priority to give more control over the outcome of computation.

The integration of structure and ornamentation as we can find it in Gothic stained glass windows seems to be promising in this regard for further research: In Gothic rose windows different figures allow to integrate structure through scaling and further subdivision. Ornament operates as structure and structure operates as ornament (L. Spuybroek, 2011). This supports the aesthetic experience of colored light.

\section{Acknowledgments}

The Swarovski diagrams shown in Figure 1 have been developed in a R\&D studio coordinated by L. Spuybroek and D. Baerlecken and prepared by students M. Ford and S. Sauer.

\section{References}

Cohen-Steiner, D., Alliez, P., and Desbrun, M. , Variational shape Approximation, ACM Transactions on Graphics, 23(3), Aug. 2004, pp. 905-914.

Glymph, J., Shelden, D., Ceccato, C., Mussel, J. and Schober, H. (2002). A Parametric Strategy for Freeform Glass Structures Using Quadrilateral Planar Facets, Thresholds - Design, Research, Education and Practice, in the Space Between the Physical and the Virtual ,Proceedings of the 2002 Annual Conference of the Association for Computer Aided Design In Architecture

Koch, R. (1975). Glass as Ornament: From Richardson to Wright, Record of the Art Museum, Princeton University, Vol. 34, No. 2, Aspects of the Artsand Crafts Movement in America (1975), pp. 28-35

Manahl, Markus; Milena Stavric, Albert Wiltsche (2012) Ornamental Discretisation of Free-form Surfaces, International Journal of Architectural Computing vol. 10 - no. 4, 595-612

Pottmann, H., Liu, Y., Wallner, J., Bobenko, A., Wang, W., (2007). Geometry of multi-layer freeform structures for architecture: efficient light transport in refractive objects, ACM Transactions on Graphics (TOG) v26 n3, 65-es

Spuybroek, L. (2011). Sympathy of things : Ruskin and the ecology of design. [Rotterdam] New York, NY: V2 Publishing : NAi Publishing.

Wenping, W.,Liu, Y., Yan, D., Ruotian, B., Sun, F., (2008). Hexagonal Meshes with Planar Faces, Technical Report, TR-2008-13, Department of Computer Science, The University of Hong Kong

Zimmer H., Campen M, Herkrath R and Kobbelt L, (2013), "Variationa Tangent Plane Intersection for Planar Polygonal Meshing" in Advances in Architectural Geometry 2012, eds L Hesselgren, S Sharma, J Wallner, N Baldassini, P Bompas and J Raynaud, Springer Vienna, Vienna, pp.319-332. 